\title{
Sequencing the CHO DXB11 genome reveals regional variations in genomic stability and haploidy
}

\author{
Christian Schrøder Kaas ${ }^{1,2,3^{*}}$, Claus Kristensen ${ }^{4}$, Michael J Betenbaugh ${ }^{3}$ and Mikael Rørdam Andersen ${ }^{2}$
}

\begin{abstract}
Background: The DHFR negative CHO DXB11 cell line (also known as DUX-B11 and DUKX) was historically the first $\mathrm{CHO}$ cell line to be used for large scale production of heterologous proteins and is still used for production of a number of complex proteins.

Results: Here we present the genomic sequence of the CHO DXB11 genome sequenced to a depth of 33x. Overall a significant genomic drift was seen favoring GC $\rightarrow$ AT point mutations in line with the chemical mutagenesis strategy used for generation of the cell line. The sequencing depth for each gene in the genome revealed distinct peaks at sequencing depths of $0 \mathrm{x}, 16 \mathrm{x}, 33 \mathrm{x}$ and 49x coverage corresponding to a copy number in the genome of $0,1,2$ and 3 copies. This indicate that $17 \%$ of the genes are haploid revealing a large number of genes which can be knocked out with relative ease. This tendency of haploidy was furthermore shown to be present in eight additional analyzed CHO genomes (15-20\% haploidy) but not in the genome of the Chinese hamster. The dhfr gene is confirmed to be haploid in CHO DXB11; transcriptionally active and the remaining allele contains a G410C point mutation causing a Thr137Arg missense mutation. We find $\sim 2.5$ million single nucleotide polymorphisms (SNP's), 44 gene deletions in the CHO DXB11 genome and 9357 SNP's, which interfere with the coding regions of 3458 genes. Copy number variations for nine $\mathrm{CHO}$ genomes were mapped to the chromosomes of the Chinese hamster showing unique signatures for each chromosome. The data indicate that chromosome one and four appear to be more stable over the course of the $\mathrm{CHO}$ evolution compared to the other chromosomes thus might presenting the most attractive landing platforms for knock-ins of heterologous genes.
\end{abstract}

Conclusions: Our studies reveal an unexpected degree of haploidy in CHO DXB11 and CHO cells in general and highlight the chromosomal changes that have occurred among the $\mathrm{CHO}$ cell lines sequenced to date.

Keywords: Copy number variations (CNVs), CHO DXB11, CHO cells, C. griseus, Single nucleotide polymorphisms (SNPs)

\section{Background}

The global market for biopharmaceuticals is currently 140 billion USD of which the majority of proteins requiring post-translational modifications are produced in Chinese Hamster Ovary (CHO) cells [1]. CHO cells have a long history as a production organism in industry due to their ability to grow in suspension without serum and to be scalable to large production volumes. Furthermore,

\footnotetext{
* Correspondence: csrk@novonordisk.com

'Mammalian Cell Technology, Global Research Unit, Novo Nordisk A/S, A9.2.36, Novo Nordisk Park, 2760, Måløv, Denmark

${ }^{2}$ Network Engineering of Eukaryotic Cell Factories, Technical University of Denmark, Kgs Lyngby, Denmark

Full list of author information is available at the end of the article
}

$\mathrm{CHO}$ cells are able to produce proteins with a glycosylation pattern similar to that of humans [2] and are not infected by a wide range of viruses dangerous to humans [3]. So far more than 40 biopharmaceuticals including monoclonal antibodies, hormones, cytokines and blood-coagulation factors have been produced in $\mathrm{CHO}$ cells.

The $\mathrm{CHO}$ cell line was originally isolated in 1957 by T. Puck [4] and ten years later the $\mathrm{CHO}-\mathrm{K} 1$ cell line was derived from this ancestral host [5]. In order to facilitate creation of stable cell lines producing a gene of interest a selection system was needed. The CHO DXB11 cell line was created with the goal of developing a stable $\mathrm{CHO}$ cell line with a DHFR negative phenotype as DHFR can catalyze the conversion of dihydrofolic acid to 
tetrahydrofolic acid - an essential cofactor carrier. CHO$\mathrm{K} 1$ cells were first exposed to a round of random chemical mutagenesis using Ethyl methanesulfonate (EMS) to generate the UKB25 cell line (dhfr+/dhfr-) [6] followed by a second round of mutagenesis using $\gamma$-radiation before isolation of the CHO DXB11 cell line (dhfr-/dhfr-) [7]. The DXB11 cell line was not mentioned by name in the original paper [6] and the name was not published until 1982 where the gene structure was more thoroughly investigated [7]. During the period between these two papers other laboratories used the cell line under the names CHO K1 DUX-B11 [8] and DUKX-CHO [9], explaining the origin of other names commonly used to describe the CHO DXB11 cell line. Further details concerning the clonal history of the $\mathrm{CHO}$ cell lines can be found in a recent review [10]. Historically, CHO DXB11 was the first $\mathrm{CHO}$ host cell for large scale production of a protein product (human tissue plasminogen activator $[10,11]$ ) and it is still being used for production of several protein products on the market.

Recently the genomic sequence of the Chinese hamster (C. griseus) $[12,13]$ and seven $\mathrm{CHO}$ cell lines [12] were released making genomic comparisons of $\mathrm{CHO}$ cells possible for the first time. The first attempt to analyze the genomic information of the CHO DXB11 cell line was done in 2005 when Wlaschin et al. extracted 4608 expressed sequencing tags from CHO DXB11 RNA in order to create a $\mathrm{CHO}$ specific cDNA microarray [14]. This work furthermore lead to sequencing of the $\mathrm{CHO}$ mitochondrial genome. A 1x coverage of the genome of a CHO DXB11 transfectant producing human secreted alkaline Phosphatase was released back in 2011 [15] the same year as the CHO-K1 ATCC sequence was made public [3]. They furthermore reported that the $d h f r$-gene was detected albeit showing low coverage.

In this work, the genome of the CHO DXB11 cell line was sequenced with the goal of making this genome publicly available alongside the list of previously sequenced $\mathrm{CHO}$ genomes [12]. The genome was analyzed in order to validate the genomic cause of the DHFR negative phenotype of the cell line and the overall genome composition was compared to the currently sequenced $\mathrm{CHO}$ genomes. We found unique patterns for the evolution of each of the chromosomes from the Chinese hamster to each of the $\mathrm{CHO}$ cell lines and a surprising degree of haploidy.

\section{Results}

Sequencing depth per gene predicts gene haploidy and polyploidy

In order to gain insight into the genotype of the industrially relevant cell line CHO DXB11, as well as addressing some of the genomic consequences associated with creating a stable $\mathrm{CHO}$ transfectant the two genomes were sequenced. Genomic DNA was extracted from adherently growing CHO DXB11 cells as well as F435 cells, which is CHO DXB11 cells transfected with the gene encoding Coagulation Factor VIII [16] and subsequently adapted to suspension culture growth. Both cell lines were sequenced using the Illumina HiSeq 2000 platform yielding 0.5 bn paired reads for CHO DXB11 and 0.3 bn reads for F435. The reads were aligned to the Chinese hamster genome with a median depth of $33 x$ and $17 x$ respectively. The sequencing depth in CHO DXB11 for each of the 20661 genes found in the C. griseus genome were calculated and plotted, showing distinct peaks at sequencing depths of $0 x, 16 x, 33 x$ and $49 x$ coverage corresponding to a copy number in the genome of $0,1,2$ and 3 copies (Figure 1A). The dhfr gene was found at a depth of 2.19 in the CHOK1 ATCC genome, 0 in the CHO DG44 genome, 1.29 in the F435 genome and 1.06 in the CHO DXB11 genome in accordance with one allele being lost by gamma radiation in CHO DXB11 (L.A. Chasin, personal communication). Seven other genes found flanking $d h f r$ on the same scaffold are also seen to be present in only one copy in the genomes of CHO DXB11 and F435. The dhfr gene from the remaining allele is found to be transcribed as seen from RNA sequencing data from F435 (Figure 1B) but contain a homozygous G410C point mutation located in the $d h f r$ coding region causing a Thr137Arg missense mutation. This threonine is conserved from C. elegans to mouse, rat, hamster and human. In the crystal structure of murine DHFR [17] (96.3\% similarity) the threonine is found in the cleft binding dihydrofolic acid right next to the active site, thus supporting the hypothesis that this mutation is able to effectively inactivate DHFR. In the process of deleting dhfr in the CHO DG44 genome it is found that four of the flanking genes were deleted (Zfyve16, Fam151b, Ankrd34b and Msh3) (Figure 1B).

\section{A significant drift in single nucleotide polymorphisms is observed}

In addition to the single nucleotide polymorphism (SNP) found in the $d h f r$ gene, a total of 2,496,390 SNPs were found in the CHO DXB11 genome when aligned to the C. griseus genome (Table 1). For the CHO-K1 ATCC genome a higher total number of SNPs were detected but more SNPs were found in the coding regions of the $\mathrm{CHO}$ DXB11 genome (Table 1). 91\% of the mutations interfering with translation in $\mathrm{CHO}-\mathrm{K} 1 \mathrm{ATCC}$ were also found in CHO DXB11. All SNPs found in CHO-K1 ATCC and CHO DXB11 genes are listed in Additional file 1: Tables S3 and S4.

By comparing all the SNPs from the available $\mathrm{CHO}$ genomes to that of $C$. griseus a significant drift favoring $\mathrm{GC} \rightarrow \mathrm{AT}$ point mutations is evident for the two $\mathrm{CHO}$ DXB11 cell lines compared to the four sequenced CHO-K1 cell lines (Figure 1C), probably caused by the chemical mutagen used in the creation of the CHO DXB11 


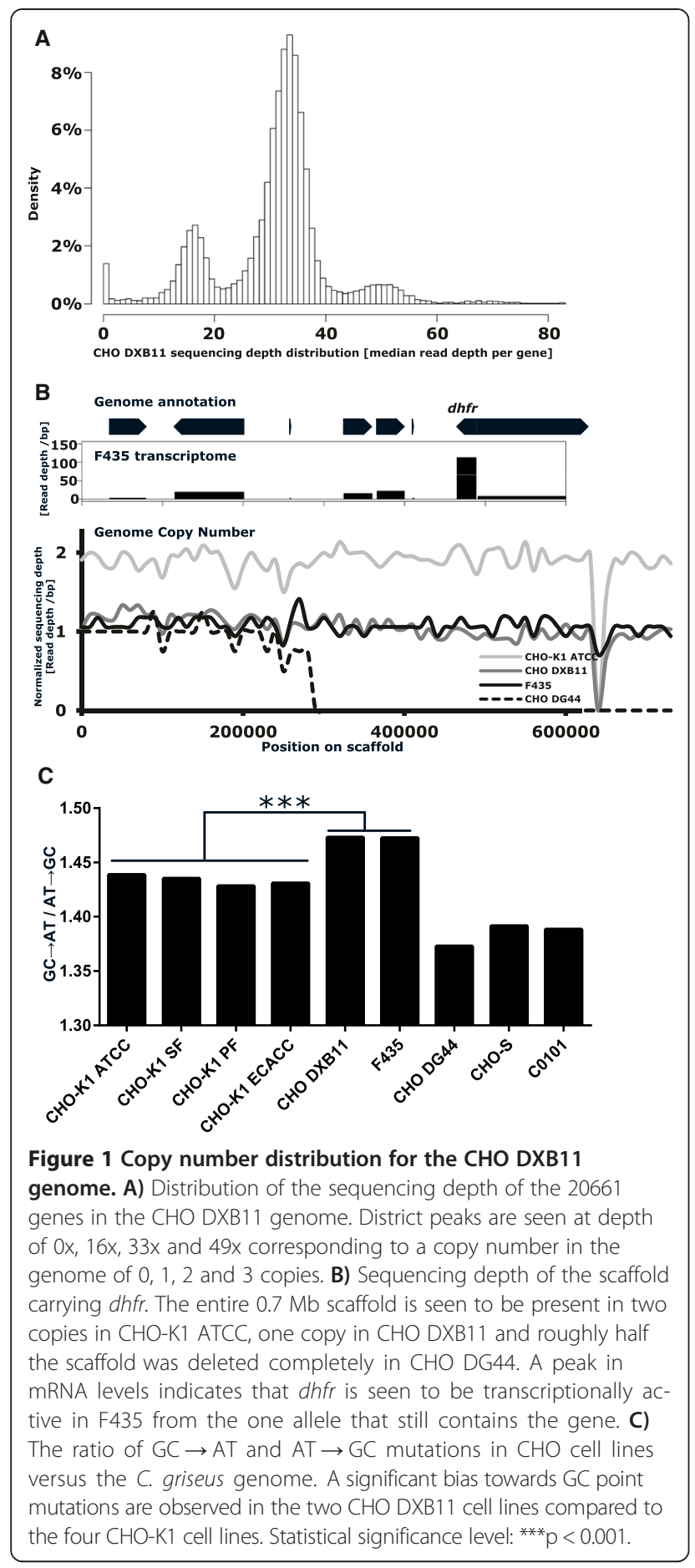

cell line. However, different SNP biases were seen for CHO DG44 and CHO-S/C0101 respectively probably due to the distinct evolution of these cell lines (Figure 1C).

\section{Copy number variation signature is chromosome dependent}

By comparing the sequencing read depth for each gene from the CHO-K1 ATCC to the CHO DXB11 genome,
Table 1 Overview of SNPs and indels in the CHO DXB11 and CHO-K1 ATCC genome

\begin{tabular}{lll}
\hline & CHO K1 ATCC & CHO DXB11 \\
\hline SNPs & $2,527,490$ & $2,496,390$ \\
Intronic SNPs & 639,171 & 636,613 \\
SNPs in CDS regions & 19,096 & 21,142 \\
SNPs missense/nonsense & 8,195 & 9,357 \\
Indels & 341,848 & 315,422 \\
Indels in CDS regions & 211 & 259 \\
Frameshifting indels & 170 & 197 \\
\hline
\end{tabular}

it is seen that $96 \%$ of the genes are found in similar depths in both cell lines as expected, following the diagonal of the plot in Figure 2. No genes are observed to be deleted in the CHO-K1 ATCC genome but present in the CHO DXB11 genome as expected due to the origin of the CHO DXB11 cell line from CHO-K1. However, a total of 506 genes are seen to have reduced copy numbers in the CHO DXB11 genome compared to the CHO-K1 ATCC genome, whereas 389 genes have increased copy number. The copy number data for the CHO-K1 ATCC and the CHO DXB11 genome were furthermore mapped to the individual chromosomes of $C$. griseus (Figure 2) revealing a unique signature for each chromosome differing from the signature of the genome as a whole. It was not possible to separate chromosome nine from chromosome ten when sequencing the $C$. griseus genome and for this reason these two are listed together [13]. On chromosome two, where $d h f r$ is situated, 89 genes have been reduced from diploid to haploid. Reductions are furthermore seen on chromosome five, six and seven. On chromosome three 158 genes are found to be triploid in CHO DXB11 versus diploid in CHO-K1 ATCC.

By estimating the extent of copy number variations (CNVs) between the currently sequenced $\mathrm{CHO}$ cell lines, a phylogenetic tree can be drawn, which accurately recapitulates the overall cell line history [10] (Figure 3). A heat map showing the extent of genes found to have reduced copy numbers between the different cell lines reveal specific patterns for each chromosome (Figure 4). From this, it can be seen that each chromosome is shown to have evolved differently across the cell lines and exhibits unique patterns. On chromosome $9 / 10, \sim 70 \%$ of the genes have been reduced from diploid in $C$. griseus to haploid in all cell lines except for C0101, CHO-S and F435 where only $\sim 50 \%$ of the genes are haploid (Additional file 2: Figure S3). Chromosome $\mathrm{X}$ is seen to contain only 9\% haploid genes in CHO-S whereas $\sim 70 \%$ of the genes on this chromosome are found to be haploid in $\mathrm{CHO}$ DG44. Chromosome five appears to have undergone changes especially in F435 (amplification) and in DG44 (amplification of some genes and reduction of others) 

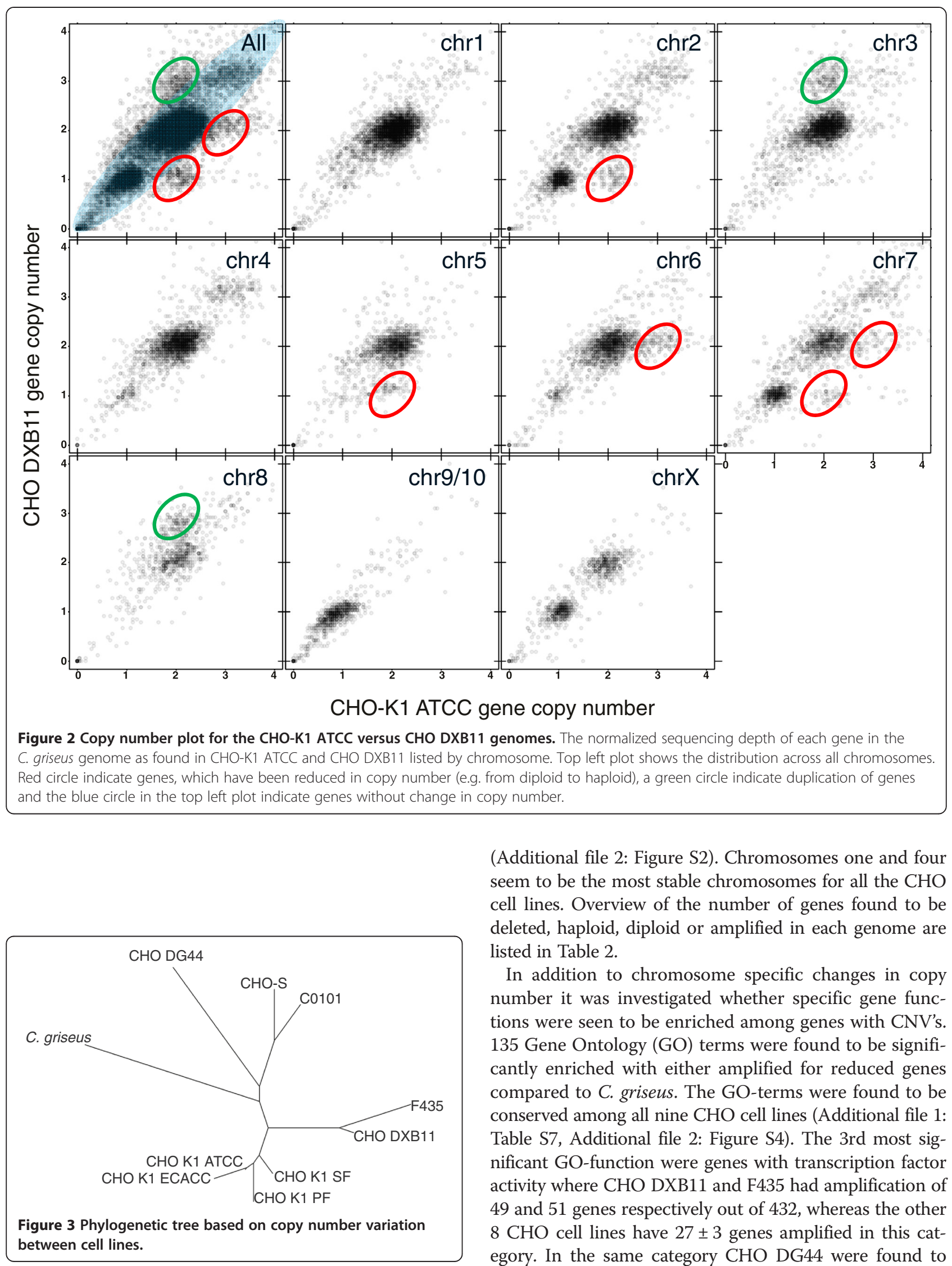

(Additional file 2: Figure S2). Chromosomes one and four seem to be the most stable chromosomes for all the $\mathrm{CHO}$ cell lines. Overview of the number of genes found to be deleted, haploid, diploid or amplified in each genome are listed in Table 2.

In addition to chromosome specific changes in copy number it was investigated whether specific gene functions were seen to be enriched among genes with CNV's. 135 Gene Ontology (GO) terms were found to be significantly enriched with either amplified for reduced genes compared to C. griseus. The GO-terms were found to be conserved among all nine $\mathrm{CHO}$ cell lines (Additional file 1: Table S7, Additional file 2: Figure S4). The 3rd most significant GO-function were genes with transcription factor activity where CHO DXB11 and F435 had amplification of 49 and 51 genes respectively out of 432 , whereas the other $8 \mathrm{CHO}$ cell lines have $27 \pm 3$ genes amplified in this category. In the same category CHO DG44 were found to 


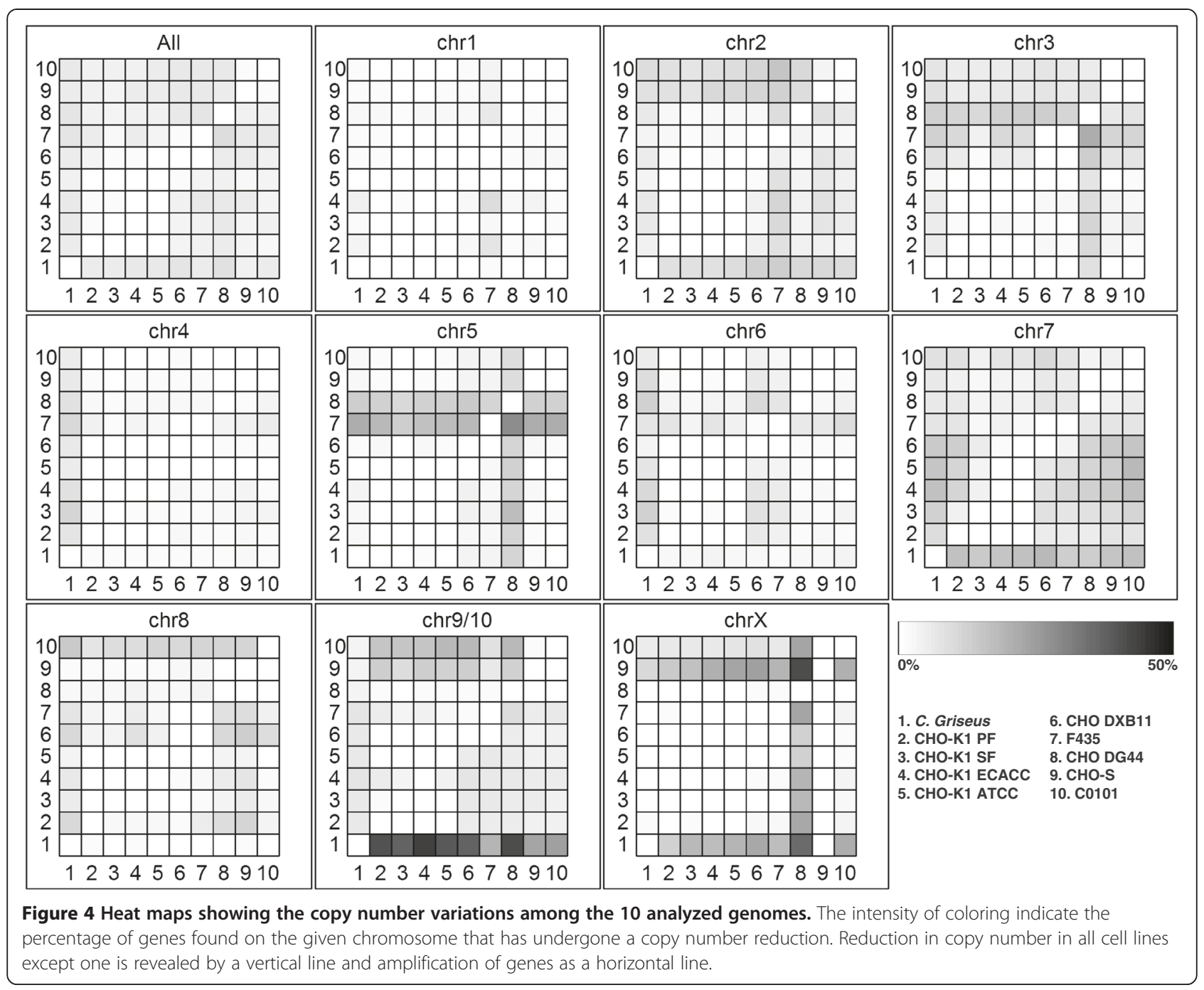

Table 2 Overview of copy number estimation in the sequenced cell lines

\begin{tabular}{lllll}
\hline & $\mathbf{C N}=\mathbf{0}$ & $\mathbf{C N}=\mathbf{1}$ & $\mathbf{C N}=\mathbf{2}$ & $\mathbf{C N}>\mathbf{2}$ \\
\hline C. griseus & 0 & 0 & 20,661 & 0 \\
CHO-K1 ATCC & 30 & 3,773 & 15,305 & 1,553 \\
CHO-K1 ECACC & 57 & 4,039 & 13,310 & 3,255 \\
CHO-K1 PF & 54 & 3,356 & 15,059 & 2,192 \\
CHO-K1 SF & 48 & 3,073 & 15,453 & 2,087 \\
CHO DXB11 & 44 & 3,586 & 15,267 & 1,764 \\
F435 & 53 & 3,888 & 14,306 & 2,414 \\
CHO DG44 & 62 & 4,219 & 13,967 & 2,413 \\
CHO-S & 47 & 3,024 & 15,603 & 1,987 \\
C0101 & 37 & 3,544 & 15,088 & 1,992 \\
\hline
\end{tabular}

have 36 genes which had been reduced in copy number where the remaining eight cell lines had $16 \pm 2$ genes reduced.

\section{Discussion}

The genetic drift of SNPs from C. griseus to $\mathrm{CHO}$ DXB11 exhibited a significant disparity compared to the drift from C. griseus to the four $\mathrm{CHO}-\mathrm{K} 1$ cell lines. The drift can likely be explained by the fact that the $\mathrm{CHO}$ DXB11 cell line was treated with the chemical mutagen EMS. The ethyl group of EMS is able to alkylate guanine forming O-6-ethylguanine which during replication commonly is paired with thymine and not cytosine $[18,19]$. Thus, an increased number of $\mathrm{GC} \rightarrow \mathrm{AT}$ mutations are expected and found in the genome of this cell line. It was seen that dhfr contained a missense mutation as well as the loss of an allele. These findings confirm observations by Lawrence Chasin in 1982 (personal communication, unpublished results). The threonine on position 137, which is mutated into an arginine in DXB11, is not found 
in any of the annotated domains of the protein but is found to be highly conserved. The amino acid is located close to the active site and it can thus be hypothesized that the polar arginine is able to interfere with the structure of the binding cleft leading to inactivation of the enzyme. Due to the fact that only one allele of $d h f r$ has been deleted and the other is transcriptionally active, it should be possible to find revertants as background in a transfection experiment. Indeed, revertants have been detected albeit at a frequency less than 10e-8 (L.A. Chasin, personal communication).

SNPs are the most frequent type of genetic polymorphism found when resequencing genomes from a common ancestor [20] and single mutations in the coding regions can result in significant changes for the phenotype of the cell lines. For sequenced diploid genomes without known and validated SNPs, it has previously been seen to filter out SNPs with a depth less than half the mean depth of the genome [12]. But in the case of CHO DXB11 (or any of the other sequenced $\mathrm{CHO}$ cell lines) that practice would result in SNPs found in most haploid genes (17\% of the genes) to be remain undetected. For that reason a more lenient filter has to be applied in the current study for homozygous SNPs, which can be found in the haploid genes, and a more stringent filter requiring higher depth for heterozygous SNPs. The need to check for mutations in the relevant cell line before designing a laborious knock down, knockout or PCR primer solely based on the C. griseus genome sequence is highlighted by the 9357 SNPs, which were found in the CHO DXB11 genome within the coding regions of 3458 genes. All genes containing SNPs as well as genes containing indels for CHOK1 ATCC and CHO DXB11 are listed in Additional file 1: Table S2. SNPs located in the other sequenced cell lines are not included due to the fact that the current lower sequencing depth of these resulted in a $\sim 20 \%$ probability of a correct SNP call versus $>99 \%$ in CHO DXB11 and K1 ATCC.

The copy number of a gene has previously been found to correlate well with the sequencing depth $[21,22]$ and this correlation was used to determine the copy number of the 20661 genes both in the C. griseus genome and in the nine sequenced $\mathrm{CHO}$ cell lines. Based on analysis of the sequencing depth per gene it was found that only wild type $C$. griseus had a single peak centered on a copy number of two whereas the sequenced $\mathrm{CHO}$ cell lines derived from this organism show distinct peaks revealing large number of genes only present in one copy or amplified to three or more copies. Interestingly, the data indicates that $15-20 \%$ of the genes found in the nine $\mathrm{CHO}$ cell lines sequenced to date are haploid. Historically, the $\mathrm{CHO}$ cells were often regarded functionally haploid at many genetic loci making these cell lines ideal for investigation of molecular functions in a eukaryotic cell model [14,23,24].
This current data explain this perceived haploid phenotype and this knowledge can also be advantageous for choosing a knock-out target in a specific pathway or for elucidating target region for a knock in. As chromosome one and four have the lowest level of CNVs across the nine $\mathrm{CHO}$ cell lines, some of which have encountered heavy mutation pressure over a long period of time [10], these chromosomes may be considered attractive landing platforms for knock-ins of heterologous genes.

Based on the current sequencing data from CHO DXB11 and available data from other $\mathrm{CHO}$ cell lines a phylogenetic tree was created based on the copy variations between the cell lines. The tree reflects the clonal history of the cell lines (see recent review for details [10]) and correlates well with a phylogenetic tree recently published based on SNPs [12]. The CHO-K1 cell lines lie in one cluster neighboring the CHO DXB11 cluster and distant from the CHO DG44 and $\mathrm{CHO}-\mathrm{S} / \mathrm{C0101}$ branches. The clonal history of the F435 cell line by transfection of CHO DXB11 is apparent from the phylogenetic tree as F435 emerges out of the same branch as CHO DXB11 (Figure 3). Nonetheless, a total of 907 genes are found to have undergone a CNV in the process from transfection of a pool of CHO DXB11 cells, amplification of the insert and subsequent adaptation to suspension culture growth.

To give a more precise estimate of the genomic differences the sequencing depth across the coding regions of the genes (1.7 kb on average) were measured. This allowed for normalization using hundreds of sequencing reads compared to looking at SNPs only supported by a dozen or so reads at most. Furthermore, the coding DNA sequences (CDS's) are the most uniquely defined elements of the genome. For this reason, assessment of the CNVs are able to provide a more detailed look into the cell lines even when only very low sequencing depths are available. This is highlighted by the clustering of the $\mathrm{CHO}-\mathrm{K} 1$ cell lines closely together, even though CHO-K1 ATCC has been sequenced to a depth of $45 \mathrm{x}$ which is $\sim 6 \mathrm{x}$ that of the other three $\mathrm{CHO}-\mathrm{K} 1$ cell lines and C0101 is sequenced to a depth $3 x$ that of CHO-S.

Each chromosome showed a distinct signature of CNVs giving for the first time an insight into $\mathrm{CHO}$ chromosomal genome stability from next-generation sequencing data. In the future this method could be used in combination with FISH to validate hypotheses on e.g. the range of genetic reductions and rearrangements on a particular chromosome (e.g. chromosome two containing $d h f r$ ) from CHO-K1 ATCC to CHO DXB11. The method also revealed a large number of haploid genes on chromosome $9 / 10$ which seem to have been reduced in the earliest $\mathrm{CHO}$ cell lines prior to evolving into the cell lines sequenced today. Some of these mutations might have been critical for establishing the independent immortal first $\mathrm{CHO}$ cell lines. 
Each rearrangement event that has occurred in the evolution from CHO-K1 to CHO DXB11 may have had an impact on a multitude of genes as seen by all eight genes on the scaffold holding $d h f r$ were found to be haploid (Figure 1B) but probably caused by one single deletion event during UV radiation. Therefore, the number of genes with altered CNVs is no true indication of the number of genomic rearrangements that has occurred as one large rearrangement events could impact dozens of genes. Due to the short lengths of the genomic scaffolds in the current versions of the C. griseus and $\mathrm{CHO}-\mathrm{K} 1$ genomes, it is not yet feasible at this time to piece together the rearrangement history of the $\mathrm{CHO}$ cell lines, but 3rd generation sequencing could permit the construction of a $C$. griseus genome with a reduced number of scaffolds [25]. Once a more complete scaffold is available, CNV data could be used to make genomic based chromosomal maps which currently are only done using FISH [26-28].

With improved genome constructions possible in coming years, it will be informative to elucidate more detailed genomic differences between CHO DG44 and $\mathrm{CHO}$ DXB11 as they are the two DHFR negative $\mathrm{CHO}$ cell lines most widely used today. As described earlier a comparison of found SNPs is not practical with the available data but it is seen from the copy numbers that 4219 genes in CHO DG44 are haploid versus 3586 in CHO DXB11. In addition, there are 44 deleted genes in CHO DXB11 versus 62 in CHO DG44. This difference can be ascribed to the harsh UV treatment that the cells were exposed to in the process of creating the CHO DG44 cell line compared to the relatively mild UV treatment of the CHO DXB11 cell line. The availability of additional sequence information from CHO-S and CHO DG44 among other would greatly improve the possibilities for comparing the genomic differences across a wider range of different $\mathrm{CHO}$ cell lines in the coming decades. This comparison could be highly informative about the evolutional path and diversity that exists across $\mathrm{CHO}$ cell hosts.

Analysis for enrichment of GO-terms revealed that a large portion of the changes in e.g. transcription factor copy number must have occurred in the early $\mathrm{CHO}$ cell. It appears that CHO DXB11 and F435 have further amplified 20 transcription factors and CHO DG44 has reduced approximately the same number. Further studies of the transcriptome and proteome should be able to reveal the effects of these genomic changes and link differences in genotype and phenotype.

\section{Conclusions}

In this work we have described the full genome sequencing of CHO DXB11 including SNPs and CNVs which differ between this cell line and the other $\mathrm{CHO}$ genomes that have been sequenced to date. The DHFR negative phenotype of the cell line was verified based on the lack of one allele and a missense mutation in the other transcriptionally active allele. The analysis of the CNVs revealed a large number of genes that were found to be haploid in the $\mathrm{CHO}$ cell lines which is important for correct SNP detection and detection strategy for knock out verification. It furthermore revealed unique patterns for the evolution of each of the chromosomes from the Chinese hamster to each of the sequenced $\mathrm{CHO}$ cell lines with chromosome one and four showing the lowest level of change.

\section{Methods}

\section{Cell culture and genome extraction}

CHO DXB11 cells were thawed from an in-house master cell bank. The cell bank was generated in 2000 from a vial of CHO DXB11 from L.A. Chasin, Columbia University. The cells were passaged in alpha MEM media with $10 \%$ FBS, 1\% NEAA, 1\% P/S. A second in-house suspension culture adapted $\mathrm{CHO}$ DXB11 cell line transfected with a plasmid encoding coagulation factor VIII coupled to $d h f r$ was grown in HyClone CDM4CHO media supplemented with $1 \%$ Penicillin/Streptomycin and 100 nM MTX. Genomic DNA from both cell lines was extracted from 2 mio cells using DNeasy Blood \& Tissue Kit (Qiagen) following manufacturer's instructions. gDNA library and next-generation sequencing were performed by AROS Applied Biotechnology (Aarhus, Denmark) in a Illimina Hisq 2000 system for paired-end sequencing.

\section{NGS data treatment}

The FASTQC tool (www.bioinformatics.bbsrc.ac.uk/projects/ fastqc/) was used to evaluate the quality of the fastq files before and after treatment. The FASTX Toolkit (http:// hannonlab.cshl.edu/fastx_toolkit/) was used to remove the adaptamers (fastx_trimmer) and trim the ends for bps with a quality score lower than 20 (fastq_quality_trimmer). An in-house algorithm was used to intersect the read-pairs after quality trimming. The reads were aligned to the C. griseus genome (downloaded from Genbank as assembly GCF_000419365.1) using BWA (version 0.6.2). The RealignerTargetCreator from GATK (version 1.6) was used to realign the reads in problematic regions and duplicate reads were removed using Picard MarkDuplicates (http://picard.sourceforge.net/).

The depth of reads at each position on the genome for identification of deleted genes was calculated using genomeCoverageBed from BEDTools (version 2.16.2). The depth for each gene was found by extracting the depth for each position in the coding region subsequently calculating the median using a custom script. The depths of each gene were normalized by $0.5 \mathrm{x}$ the median depth of all genes. The data were analyzed in $R$ [29]. The depths are listed in Additional file 1: Table S5 and an overview of all genes in C. griseus with Genbank IDs, GC content and 
chromosome number is listed in Additional file 1: Table S6. The aligned reads from CHO DXB11 to the $C$. griseus genome was uploaded to the SRA under experiment ID: SRX689758. For direct download of raw reads from all public $\mathrm{CHO}$ cell lines currently in the SRA see Additional file 1: Table S1.

\section{SNP calls}

SNPs were detected by samtools mpileup and bcftools. SNPs present in the coding region were found using CLC genomic workbench (CLC Bio, version 7). Haploid SNPs were detected by the filter: A minimum depth of $0.25 \mathrm{x}$ the median depth measured in the CDS regions, $90 \%$ of the reads calling the SNP had to differ from the reference sequence. Heterologous SNPs: A minimum depth of $0.75 x$ the median depth measured in the CDS regions, a minimum of $40 \%$ of the reads should agree with the reference and $40 \%$ with the called allele. All SNPs interspaced by less than 5 bp to another SNP or an indel were filtered away as well as SNPs found when aligning the raw C. griseus reads to the C. griseus genome. Mutational bias was calculated by extracting reference and non-reference bases from the filtered SNP files and calculating the occurrence of different nucleotide transitions. Significance of bias were calculated as standard student $\mathrm{t}$-test assuming equal variance comparing the observations for $\mathrm{GC} \rightarrow \mathrm{AT} / \mathrm{AT} \rightarrow \mathrm{GC}$ for $\mathrm{CHO}-\mathrm{K} 1 \mathrm{PF}, \mathrm{CHO}-\mathrm{K} 1$ SF, CHO-K1 ECACC and CHO-K1 ATCC versus CHO DXB11 and F435.

\section{Phylogenetic tree based on CNV data}

Raw sequencing reads from $\mathrm{CHO}-\mathrm{K} 1$ [3] and other $\mathrm{CHO}$ cell lines [12] were downloaded from the SRA (Additional file 1: Table S1). Reads were trimmed and intersected as described above and aligned to the $C$. griseus genome using BWA. Depths were estimated for each gene in each cell lined as described above and the occurrence of CNVs were estimated as the number of genes differing by more than 0.95 in normalized sequencing depth between two cell lines. A distance matrix were calculated, a phylogenetic tree was constructed using $\mathrm{R}$ with the package ape and phangorn using the neighbour joining algorithm. The tree was bootstrapped 100 times and the consensus tree was used.

\section{Gene copy number}

The absolute copy number for each gene in each cell line was calculated as above by normalizing the read depth to the median. Genes were considered to be deleted if the read depth were 0 in a given cell line but > 0.95 in C. griseus (as 159 genes were $\sim 0$ in all cell lines incl. C. griseus). Haploid: depth higher than 0 but lower than 1.3 based on local minimum in CHO DXB11 between the haploid and diploid peak. Diploid: higher than
1.3 and lower than 2.7 based on local minimum in $\mathrm{CHO}$ DXB11 between the diploid and triploid. Triploid or higher: depth higher than 2.7 .

\section{Chromosomal changes}

All genes from the $C$. griseus genome (assembly GCF_000419365.1) as listed in the genome annotation file were downloaded from Genbank. The chromosome sorted C. griseus genome was downloaded from Genbank as Accession APMK00000000. The scaffold name and chromosome number was extracted from the fasta header. All genes were blasted against the chromosome sorted genome and the best hit (cutoff E-value $=0.05$ ) was used as indicator for the chromosomal location.

\section{RNA sequencing}

In order to deduce the transcriptional activity of $d h f r$ from F435, RNA was extracted. A sample was taken 48 hours into the cultivation from $2 x 10 \mathrm{e} 6$ cells and RNA was extracted using TRIzol (Invitrogen) and the RNeasy Cleanup kit (Qiagen) following manufacturer's instructions. RNA integrity was confirmed on an Agilent 2100 Bioanalyzer using total RNA nano chips (Agilent technologies, Santa Clara, Ca, USA). RNA concentration was measured using a NanoDrop spectrophotometer (NanoDrop Technologies). Multiplexed cDNA library generation and next-generation sequencing were performed by AROS Applied Biotechnology (Aarhus, Denmark) in an Illumina Hiseq 2000 system for paired-end sequencing. The FASTQC tool (http://www. bioinformatics.babraham.ac.uk/projects/fastqc/) was used to evaluate the quality of the fastq files before and after treatment. The FASTX Toolkit (http://www.bioinformatics. babraham.ac.uk/projects/fastqc/) was used to remove the adaptamers (fastx_trimmer) and trim the ends for base pairs with a quality score lower than 20 (fastq_quality_ trimmer). An in-house algorithm was used to intersect the read-pairs after quality trimming. The reads were aligned to the CHO-K1 genome (downloaded from Genbank as assembly GCF_000223135.1) using tophat2 [30]. RNA expression from each of the genes on the NW_ 003614442.1 scaffold containing $d$ hfr was calculated using genomeCoverageBed from BEDTools (version 2.16.2) and the mean expression level was used as indicator for expression.

\section{GO-term enrichment}

A list of GO-terms associated with the $\mathrm{CHO}$ genome was downloaded from CHOgenome.org (http://www. chogenome.org/files/CHO_GO_Functions_12Sep13.txt), the list was rearranged and imported into R. Fisher's exact test were used to find GO-terms enriched for either reduced or amplified genes for each of the $\mathrm{CHO}$ cell lines compared to C. griseus by $>0.95$ difference in normalized copy number. All GO-terms, which had a p-value $<0.01$ 
in just one cell line for either amplification or reduction in $\mathrm{CN}$ were included. Data listed in Additional file 1: Table S7.

\section{Additional files}

Additional file 1: Table S1. SRA. Table S2. SNP qeneoverview. Table S3. Exon SNP's DXB11. Table S4. Exon SNP's K1ATCC. Table S5. Sequencing depth. Table S6. Cqriseus overvie. Table S7. GO-terms.

Additional file 2: Figure S1. Read depth analysis of the 20661 in the C. griseus genome. For most of the genomes distinct peaks can be seen for genes present in one, two and three copies. Shoulders can be seen in the graphs for the cell lines sequenced at a lower depth. Only one peak is seen in wild type C. griseus as expected. Figure S2. The normalized sequencing depth of each gene in F435 and CHO DG44. Top left plot shows the distribution across all chromosomes. Compared to Figure 2 this reveals a much larger difference in CN. Figure S3. Distribution of CN across chromosomes for each cell line. A) Percentage of haploid genes B) percentage of diploid genes C) Percentage of genes, which are triploid or higher. Figure S4. Significant GO-terms in correlation to changes in CN Visualization of the 135 GO-terms, which are either significantly enriched in genes with $\mathrm{CN}$ reductions or amplifcations (Fisher's exact test). GO-terms are visualized in dark blue ( $p$-value $<0.01$ ), light blue ( $p$-value $<0.05)$ or white $(p$-value $>0.05)$. Data attached in Additional file 1: Table S7.

\section{Competing interest}

The authors declare that they have no competing interests.

\section{Authors' contributions}

CSK carried out the cell work, data analysis and drafted the manuscript. CK, MBJ and MR provided guidance throughout the data analysis and manuscript formulation. All authors read and approved the final manuscript.

\section{Acknowledgements}

We would like to acknowledge Dr. Nathan Lewis for kindly providing raw reads from the C. griseus sequencing [12], Dr Lawrence Chasin for providing essential input on the history of the CHO DXB11 cell line and finally the reviewers for providing essential input for improving the paper.

\section{Author details}

${ }^{1}$ Mammalian Cell Technology, Global Research Unit, Novo Nordisk A/S, A9.2.36, Novo Nordisk Park, 2760, Måløv, Denmark. ${ }^{2}$ Network Engineering of Eukaryotic Cell Factories, Technical University of Denmark, Kgs Lyngby, Denmark. ${ }^{3}$ Chemical and Biomolecular Engineering, Johns Hopkins University, Baltimore, MD, USA. Institute of Cellular and Molecular Medicine, University of Copenhagen, Copenhagen, Denmark.

Received: 28 November 2014 Accepted: 24 February 2015

Published online: 08 March 2015

\section{References}

1. Walsh G. Biopharmaceutical benchmarks 2014. Nat Biotechnol. 2014;32:992-1000

2. Kim JY, Kim YG, Lee GM. CHO cells in biotechnology for production of recombinant proteins: current state and further potential. Appl Microbiol Biotechnol. 2012;93:917-30

3. Xu X, Nagarajan H, Lewis NE, Pan S, Cai Z, Liu X, et al. The genomic sequence of the Chinese hamster ovary $(\mathrm{CHO})-\mathrm{K} 1$ cell line. Nat Biotechnol. 2011;29:735-41.

4. Puck TT. Genetics Of Somatic Mammalian Cells: III. Long-term Cultivation Of Euploid Cells From Human And Animal Subjects. J Exp Med. 1958;108:945-56.

5. Kao FT, Puck TT. Genetics of somatic mammalian cells, VII. Induction and isolation of nutritional mutants in Chinese hamster cells. Proc Natl Acad Sci USA. 1968;60:1275-81.

6. Urlaub G, Chasin L. Isolation of Chinese hamster cell mutants deficient in dihydrofolate reductase activity. Proc Natl Acad Sci U S A. 1980;77:4216-20.
7. Graf Jr LH, Chasin LA. Direct demonstration of genetic alterations at the dihydrofolate reductase locus after gamma irradiation. Mol Cell Biol. 1982;2:93-6.

8. Gasser CS, Simonsen CC, Schilling JW, Schimke RT. Expression of abbreviated mouse dihydrofolate reductase genes in cultured hamster cells. Proc Natl Acad Sci. 1982;79(21):6522-6.

9. Kaufman RJ, Sharp P. Amplification and expression of sequences cotransfected with a modular dihydrofolate reductase complementary dna gene. J Mol Biol. 1982;159:601-21.

10. Wurm F. CHO Quasispecies - Implications for Manufacturing Processes. Processes. 2013:1:296-311.

11. Kaufman RJ, Wasley LC, Spiliotes AJ, Gossels SD, Latt S, Larsen GR, et al. Coamplification and coexpression of human tissue-type plasminogen activator and murine dihydrofolate reductase sequences in Chinese hamster ovary cells. Mol Cell Biol. 1985;5:1750-9.

12. Lewis NE, Liu X, Li Y, Nagarajan H, Yerganian G, O'Brien E, et al. Genomic landscapes of Chinese hamster ovary cell lines as revealed by the Cricetulus griseus draft genome. Nat Biotechnol. 2013;31:759-65.

13. Brinkrolf K, Rupp O, Laux H, Kollin F, Ernst W, Linke B, et al. Chinese hamster genome sequenced from sorted chromosomes. Nat Biotechnol. 2013:31:694-5.

14. Wlaschin KF, Nissom PM, Gatti MDL, Ong PF, Arleen S, Tan KS, et al. EST sequencing for gene discovery in Chinese hamster ovary cells. Biotechnol Bioeng. 2005;91:592-606.

15. Hammond S, Swanberg JC, Kaplarevic M, Lee KH. Genomic sequencing and analysis of a Chinese hamster ovary cell line using Illumina sequencing technology. BMC Genomics. 2011;12:67.

16. Thim L, Vandahl B, Karlsson J, Klausen NK, Pedersen J, Krogh TN, et al. Purification and characterization of a new recombinant factor VIII (N8). J World Federation Hemophilia. 2010;16:349-59.

17. Cody V, Pace J, Rosowsky A. Structural analysis of a holoenzyme complex of mouse dihydrofolate reductase with NADPH and a ternary complex with the potent and selective inhibitor 2,4-diamino-6-(2'-hydroxydibenz[b, f] azepin-5-yl)methylpteridine. Acta Crystallogr D Biol Crystallogr. 2008;64:977-84.

18. Heflich RH, Beranek DT, Kodell RL, Morris SM. Induction of mutations and sister-chromatid exchanges in Chinese hamster ovary cells by ethylating agents. Mutation Research/Fundamental and Molecular Mechanisms of Mutagenesis. 1982;106:147-61.

19. Flibotte S, Edgley ML, Chaudhry I, Taylor J, Neil SE, Rogula A, et al, Whole-genome profiling of mutagenesis in Caenorhabditis elegans. Genetics. 2010;185:431-41.

20. Brookes AJ. The essence of SNPS. Gene. 1999;234:177-86.

21. Alkan C, Kidd JM, Marques-Bonet T, Aksay G, Antonacci F, Hormozdiari F, et al. Personalized copy number and segmental duplication maps using next-generation sequencing. Nat Genet. 2009;41:1061-7.

22. Medvedev P, Fiume M, Dzamba M, Smith T, Brudno M. Detecting copy number variation with mated short reads. Genome Res. 2010;20:1613-22

23. Jeggo PA, Holliday R. Azacytidine-induced reactivation of a DNA repair gene in Chinese hamster ovary cells. Mol Cell Biol. 1986;6:2944-9.

24. Siminovitch $\mathrm{L}$. On the nature of hereditable variation in cultured somatic cells. Cell. 1976;7:1-11.

25. Borth N. Opening the black box: Chinese hamster ovary research goes genome scale. Pharma Bioprocessing. 2014;2:367-9.

26. Cao $Y$, Kimura S, Itoi T, Honda K, Ohtake H, Omasa T. Construction of BAC-based physical map and analysis of chromosome rearrangement in Chinese hamster ovary cell lines. Biotechnol Bioeng. 2012;109:1357-67.

27. Toledo F, Buttin G+, Debatisse M. The origin of chromosome rearrangements at early stages of AMPD2 gene amplification in Chinese hamster cells. Curr Biol. 1993;3:255-64.

28. Day J. Recombination involving interstitial telomere repeat-like sequences promotes chromosomal instability in Chinese hamster cells. Carcinogenesis. 1998;19:259-65.

29. Ihaka R, Gentleman R. R: A Language for Data Analysis and Graphics. J Computational Graph Stat. 1996;5:299-314.

30. Trapnell C, Pachter L, Salzberg SL. TopHat: discovering splice junctions with RNA-Seq. Bioinformatics. 2009:25:1105-11. 